\title{
Blood Pressure and Early Clinical Outcome Among Acute Ischemic Stroke Patients
}

\author{
Jintao Zhang, Ying Peng, Huanqing Fan, Mei Chen, Tan Xu, Yonghong Zhang
}

\begin{abstract}
Objectives: The association between blood pressure (BP) and short-term clinical outcome of acute ischemic stroke is inconclusive. We investigated the association between BP in the first 72 hours following admission and death in-hospital and neurologic deficiency at discharge among patients with acute ischemic stroke. Methods: A total of 2675 acute ischemic stroke patients confirmed by a computed tomography scan or magnetic resonance imaging were included in the present study. Blood pressure in the first 72 hours after admission and other study variables were collected for all ischemic stroke patients. Neurological functions National Institute of Health Stroke Scale (NIHSS) were evaluated by trained neurologists at discharge. The study outcome was defined as death in-hospital and neurologic deficiency (NIHSS $\geq 10$ ) at discharge. Results: Systolic and diastolic BP were significantly and positively associated with odds of study outcome in acute ischemic stroke. For example, compared to those with a systolic BP $<140 \mathrm{mmHg}$, multiple-adjusted odds ratio (95\% confidence interval) of study outcome was 3.29(1.22, 8.90) among participants with systolic BP of 180-219 mmHg, $P<0.05$; compared to those with a diastolic $\mathrm{BP}<90 \mathrm{mmHg}$, multiple-adjusted odds ratio of study outcome was $7.05(1.32,37.57)$ among participants with diastolic BP $\geq 120 \mathrm{mmHg}, P<0.05$. Conclusion: Systolic $\mathrm{BP} \geq 180$ and diastolic $\mathrm{BP} \geq 120$ were significantly and positively associated with death in-hospital or neurologic deficiency at discharge among patients with acute ischemic stroke.
\end{abstract}

RÉSUMÉ: La tension artérielle et l'issue clinique précoce chez les patients atteints d'un accident vasculaire cérébral ischémique aigu. Objectifs : L'association entre la tension artérielle (TA) et l'issue clinique à court terme suite à un accident vasculaire cérébral ischémique aigu (AVCIA) demeure confuse. Nous avons étudié, chez des patients atteints d'un AVCIA, la relation entre la TA dans les 72 premières heures après l'admission et le décès intra hospitalier et le déficit neurologique au moment du congé hospitalier. Méthode : Nous avons inclus dans cette étude 2675 patients atteints d'un AVCIA confirmé par tomodensitométrie ou imagerie par résonance magnétique. La TA dans les 72 premières heures après l'admission à l'hôpital ainsi que d'autres variables ont été recueillies pour chacun des patients. Les fonctions neurologiques (NIHSS) ont été évaluées au moment du congé hospitalier par des neurologues d'expérience. Le critère d'évaluation de l'étude était le décès hospitalier et le déficit neurologique (NIHSS $\geq 10$ ) au moment du congé. Résultats : La TA systolique et la TA diastolique étaient significativement et positivement associées à l'issue suite à un AVCIA. Ainsi, comparé à ceux dont la TA systolique était < $140 \mathrm{mmHg}$, le rapport de cotes après ajustements multiples (IC à 95\%) du critère d'évaluation était 3,29 $(1,22$ à 8,90$)$ chez les participants dont la TA systolique était de 180 à $219 \mathrm{mmHg}, \mathrm{p}<0,05$. Par rapport à ceux dont la TA diastolique était de < 90 $\mathrm{mmHg}$, le rapport de cotes après ajustement multiples du critère d'évaluation était 7,05 $(1,32$ à 37,57$)$ chez les participants dont la TA diastolique était $\geq 120 \mathrm{mmHg}, \mathrm{p}<0,05$. Conclusion : Une TA systolique $\geq 180$ et une TA diastolique $\geq 120$ étaient significativement et positivement associées au décès intrahospitalier ou au déficit neurologique au moment du congé hospitalier chez les patients atteints d'un AVCIA.

Can J Neurol Sci. 2011; 38: 225-229

Stroke is the third leading cause of death and long-term disability world wide ${ }^{1}$. The burden of stroke is high and growing in economically developing countries, including China ${ }^{2}$. Stroke accounted for $21.6 \%$ of total mortality in men and $20.8 \%$ of total mortality in women in $\mathrm{China}^{3}$. In addition, it was estimated that seven million Chinese people had prevalent stroke in $2004^{4}$. The incidence and prevalence of stroke and proportion of ischemic stroke is gradually increasing in the Chinese population ${ }^{5,6}$. Prospective cohort studies and clinical trials have documented that high blood pressure (BP) is the most important modifiable risk factor for the incidence of stroke ${ }^{7-9}$. However, the relationship between acute phase BP and clinical outcome among acute ischemic stroke patients is much less certain ${ }^{10,11}$.
Some observational studies have suggested that elevated BP is associated with poor outcome for acute ischemic stroke patients $^{12-15}$, whereas others have showen a 'U-shaped' relationship between acute phase BP and clinical outcome ${ }^{16-19}$.

From the Department of Neurology (JZ, HF), The 88th Hospital of PLA, Taian, Shandong Province; Department of Epidemiology (YP, MC, TX, YZ), School of Radiation Medicine and Public Health, Medical College of Soochow University, Suzhou, China.

Received June 21, 2010. Final Revisions Submitted September 15, 2010. Correspondence to: Yonghong Zhang, Department of Epidemiology, School of Radiation Medicine and Public Health, Medical College of Soochow University, 199 Renai Road, Industrial Park District, Post Code: 215123, Suzhou, China. 
Table 1: Baseline characteristics of ischemic stroke patients with Death or NIHSS $\geq 10$ or NIHSS $<10$

\begin{tabular}{llll}
\hline Variables & NIHSS $<10$ & Death/ NIHSS $\geq 10$ & $P$-value \\
\hline $\mathrm{N}$ & 2470 & 205 & - \\
Age, $(\bar{x} \pm S)$ & $64.2 \pm 11.9$ & $65.7 \pm 11.5$ & 0.087 \\
Men, $\mathrm{n}(\%)$ & $1560(63.2)$ & $127(62.0)$ & 0.731 \\
Cigarette smoking, $\mathrm{n}(\%)$ & $615(25.4)$ & $50(20.0)$ & 0.904 \\
Alcohol drinking, $\mathrm{n}(\%)$ & $598(24.7)$ & $43(16.4)$ & 0.371 \\
High TC, $\mathrm{n}(\%)$ & $296(12.1)$ & $29(10.8)$ & 0.305 \\
High TG, $\mathrm{n}(\%)$ & $366(14.7)$ & $27(10.3)$ & 0.685 \\
High LDL-C, $\mathrm{n}(\%)$ & $220(8.9)$ & $31(12.6)$ & 0.030 \\
Low HDL-C, $\mathrm{n}(\%)$ & $714(28.6)$ & $45(17.6)$ & 0.154 \\
Hyperglycemia, $\mathrm{n}(\%)$ & $792(34.0)$ & $116(49.8)$ & $<0.001$ \\
History of hypertension, $\mathrm{n}(\%)$ & $1515(61.8)$ & $119(48.6)$ & 0.264 \\
History of diabetes, $\mathrm{n}(\%)$ & $359(14.6)$ & $28(12.2)$ & 0.621 \\
History of coronary heart disease, $\mathrm{n}(\%)$ & $401(16.3)$ & $38(18.1)$ & 0.607 \\
History of auricular fibrillation, $\mathrm{n}(\%)$ & $194(2.6)$ & $<0.001$ \\
History of rheumatic heart disease, $\mathrm{n}(\%)$ & $18(0.7)$ & $5(1.5)$ & 0.011 \\
Family history of stroke, $\mathrm{n}(\%)$ & $8(5.0)$ & 0.432 \\
Family history of hypertension, $\mathrm{n}(\%)$ & $134(5.6)$ & $7(3.0)$ & 0.213 \\
Family history of diabetes, $\mathrm{n}(\%)$ & $2(2.05)$ & 0.471 \\
Duration of hospitalization, median (interquartile), & $15(10,21)$ & $13(7,21)$ & 0.001 \\
days & & & \\
Time from onset to admission, median (interquartile), & $48(7,144)$ & $19(4,73)$ & $<0.001$ \\
hours & & & \\
\hline
\end{tabular}

\# adjusted by age, gender; High TC: Total cholesterol $\geq 6.22 \mathrm{mmol} / \mathrm{L}$; High TG: Triglyceride $\geq 2.26 \mathrm{mmol} / \mathrm{L}$; High LDL-C: Low-density lipoprotein-cholesterol $\geq 4.14 \mathrm{mmol} / \mathrm{L}$; Low HDL-C: high-density lipoproteincholesterol $<1.04 \mathrm{mmol} / \mathrm{L}$; Hyperglycemia: Fast plasma glucose $\geq 6.1 \mathrm{mmol} / \mathrm{L}$.

Several studies did not show that elevated BP related to poor outcome 20,21 .

There are currently few data on the relationship between acute phase BP and short-term clinical outcome among acute ischemic stroke patients in Chinese populations. We investigated the association between systolic and diastolic BP levels in the first 72 hours following admission and death in-hospital and neurologic deficiency among acute ischemic stroke patients.

\section{Methods}

\section{Study participants}

Acute ischemic stroke patients were recruited from four hospitals in Shandong province, China. All patients with a clinical diagnosis of acute ischemic stroke admitted to the four hospitals from 1 January 2006 to 31 December 2008 were potentially eligible for the study. However, only those cases confirmed by a computed tomography (CT) scan or magnetic resonance imaging (MRI) were included in the study. A team of investigators including neurologists reviewed the eligibility of study participants. Among 2868 acute ischemic stroke patients admitted to the four hospitals during the study period, 2675 had a CT scan or MRI confirmed ischemic stroke and were included in this analysis. A total of 193 patients were excluded from our analysis because of lack of a CT scan or MRI test. Stroke patients who were not admitted into the four hospitals including those who died outside of hospitals were not included in this analysis. This study was approved by Soochow University School of Radiation Medicine and Public Health Ethics Committee.

\section{Data collection}

Baseline data were collected within the first 24 hours of hospital admission by in-person interview with patients or their family members (if patients were not able to communicate). Data on demographic characteristics, life-style risk factors, medical history, clinical laboratory tests, and imaging data (CT and MRI) were obtained using a standard questionnaire administered by trained staff. Cigarette smokers were defined as having smoked at least one cigarette per day for one year or more. The amount and type of alcohol consumed during the past year was collected. Alcohol consumption was defined as consuming one or more alcoholic drinks per day during the last year. Blood pressure measurements were taken in the first 72 hours (one measurement every eight hours) after admission while the study participants were in the supine position, using a standard mercury sphygmomanometer according to a standard protocol ${ }^{22}$. The first and fifth Korotkoff sounds were recorded as systolic and diastolic BP, respectively.

Plasma glucose was measured using a modified hexoki-nase enzymatic method. Total cholesterol, high-density lipoprotein (HDL)-cholesterol, and triglycerides were analyzed enzymatically on a Beckman Synchron CX5 Delta Clinical System (Beckman Coulter, Inc., Fullerton, California, USA) using commercial reagents ${ }^{23}$. Low-density lipoprotein (LDL)cholesterol levels were calculated by the use of the Friede wald equation $^{24}$. If a patient died in the hospital, a study staff member recorded the death on the event form and obtained the death certificate. If a patient survived the acute stroke, the study 
neurologists conducted a comprehensive clinical evaluation using National Institutes of Health (NIH) stroke scale (NIHSS) at discharge. The study outcomes was defined as death during hospitalization and neurologic deficiency (NIHSS $\geq 10$ ) at discharge.

\section{Statistical analysis}

Patients were divided into two groups, with study outcome and without study outcome. The mean and standard deviation of continuous variables and proportion of categorical variables at hospital admission were calculated and compared between patients with study outcome and without study outcome. Patients were also grouped by BP level (systolic BP $<140$, 140-159, 160$179,180-219, \geq 220 \mathrm{mmHg}$ or diastolic $\mathrm{BP}<90,90-99,100-109$, $110-119, \geq 120 \mathrm{mmHg}$ ). Multiple logistic regression analysis was used to examine the association between BP and study outcome adjusted for age, gender, alcohol consumption, cigarette smoking, dyslipidemia, hyperglycemia and histories of hypertension, diabetes, coronary heart disease, auricular fibrillation and rheumatic heart disease, and family histories of stroke, hypertension and diabetes, and consciousness level and complications including myocardial infarction, heart failure, renal failure and pneumochysis at admission. The presence of a linear association was tested using the median systolic and diastolic BP in each of the categories as a continuous variable in the multiple logistics models. Statistical analyses were conducted using SAS statistical software (version 9.1; SAS Institute Inc, Cary, North Carolina, USA).

\section{Results}

A total of 2675 ischemic stroke patients were included in our analysis, there were 2470 patients without study outcome and 205 patients with study outcome (19 deaths and 186 patients with NIHSS $\geq 10$ ).

Table 1 presents the demographic and clinical characteristics at admission by study outcome. Those with study outcome were more likely to have high LDL-C, hyperglycemia, histories of auricular fibrillation and rheumatic heart disease. There was no significant difference in age, gender, smoking, alcohol consumption, high TC, high TG, low HDL-C, family history of stroke, family history of hypertension, family history of diabetes between patients with study outcome and without study outcome.

Participants with study outcome had higher average systolic and diastolic BP levels in the first 72 hours after admission compared with those without study outcome, but the difference did not reach statistical significance (Table 2). The median duration of hospitalization was 13 days for patients with study outcome and 15 days for patients without study outcome, respectively. The median time from onset to admission was 19 hours for patients with study outcome and 48 hours for patients without study outcome, respectively.

There were positive and significant associations between high systolic and extreme high diastolic BP levels in the first 72 hours of admission and study outcome among patients with acute ischemic stroke (Table 3). Systolic BP of 180-219 mmHg or more was associated with 3.29-fold higher odds of study outcome compared to normal systolic BP after adjustment for important covariates (there was one death among only two patients with systolic $\mathrm{BP} \geq 220 \mathrm{mmHg}$ in total paricipants). There was a trend that the risk was increased with elevated systolic BP $(P<0.05)$. Similarly, the patients with diastolic BP $\geq 120 \mathrm{mmHg}$ had more risk of study outcome compared to those with normal diastolic BP $(P<0.05)$. However, a significant association between mild high diastolic BP and risk of study outcome was not found in the analysis.

\section{Discussion}

Our study found a statistically significant association between elevated systolic ( $\geq 180 \mathrm{mmHg}$ ) and diastolic $(\geq 120$ $\mathrm{mmHg}$ ) BP in the first 72 hours of admission and early death or neurologic deficiency (NIHSS $\geq 10$ ) among acute ischemic stroke patients. In multivariate logistic analysis, we also adjusted for consciousness level and complications including myocardial infarction, heart failure, renal failure and pneumochysis at admission, which could reflect the stroke severity. These findings appear to imply that high systolic BP and extreme high diastolic BP after onset of ischemic stroke could predict a poor

Table 2: The systolic (SBP) and diastolic BP (DBP) levels adjusted by age and gender in first 72 hours after admission among ischemic stroke patients

\begin{tabular}{lccc}
\hline BP levels after admission & NIHSS $<10$ & Death/ NIHSS $\geq 10$ & $P$-value \\
\hline $\mathrm{N}$ & 2470 & 205 & - \\
\hline SBP, mean $(95 \% \mathrm{CI}), \mathrm{mmHg}$ & & & \\
Within 24 h & $145.3(144.5,146.2)$ & $148.6(145.6,151.6)$ & 0.042 \\
25-48 h & $139.9(139.1,140.7)$ & $138.5(141.7,147.2)$ & 0.002 \\
49-72 h & $139.4(138.7,140.2)$ & $143.6(136.1,141.5)$ & 0.651 \\
Average within 72 h & $141.6(140.9,142.3)$ & & 0.116 \\
& & & \\
DBP, mean (95\%CI), mmHg & & $88.7(86.8,90.5)$ & 0.255 \\
Within 24 h & $87.5(87.0,88.1)$ & $86.7(85.0,88.5)$ & 0.060 \\
25-48 h & $85.0(84.5,85.5)$ & $83.8(82.0,85.5)$ & 0.273 \\
49-72 h & $84.8(84.3,85.3)$ & $86.4(85.0,87.9)$ & 0.419 \\
Average within 72 h & $85.8(85.4,86.2)$ & \\
\hline
\end{tabular}


Table 3: Odds ratio and $95 \%$ confidence intervals of Death or NIHSS $\geq 10$ with the mean $B P$ of the first 72 hours after admission in patients with acute ischemic stroke

\begin{tabular}{lllll}
\hline BP & Unadjusted $O R(95 \% C I)$ & $P$ value & Adjusted OR $(95 \% C I)$ & $P$ value \\
\hline Systolic BP,mmHg & & & & \\
$<140$ & $1.00($ ref $)$ & & $1.00($ ref $)$ & \\
$140-159$ & $1.18(0.84,1.66)$ & 0.339 & $1.24(0.83,1.85)$ & 0.287 \\
$160-179$ & $1.28(0.78,2.08)$ & 0.331 & $1.52(0.87,2.69)$ & 0.145 \\
$180-219$ & $2.67(1.15,6.19)$ & 0.023 & $3.29(1.22,8.90)$ & 0.019 \\
$\geq 220$ & - & - & - & - \\
Trend test & $P=0.024$ & & $P=0.015$ & \\
Diastolic BP,mmHg & & & & \\
$<90$ & $1.000($ ref $)$ & 0.155 & $1.00($ ref $)$ & 0.120 \\
$90-99$ & $1.29(0.91,1.82)$ & 0.792 & $1.05(0.51,2.17)$ & 0.888 \\
$100-109$ & $0.92(0.48,1.74)$ & - & - & - \\
$110-119$ & - & 0.001 & $7.05(1.32,37.57)$ & 0.022 \\
$\geq 120$ & $8.78(2.44,31.60)$ & & $P=0.316$ & \\
Trend test & $P=0.317$ & & \\
\hline
\end{tabular}

\# adjusted for age, gender, alcohol consumption, cigarette smoking, dyslipidemia, hyperglycemia and histories of hypertension, diabetes, coronary heart disease, auricular fibrillation and rheumatic heart disease, family histories of stroke, hypertension and diabetes, consciousness state and complications at admission. SBP=systolic blood pressure; $\mathrm{DBP}=$ diastolic blood pressure.

clinical outcome. In fact, some observational studies ${ }^{14,15}$ have demonstrated a significant association of high BP with poor outcome. For example, Robinson's study ${ }^{14}$ showed that systolic $\mathrm{BP} \geq 160 \mathrm{mmHg}$ within 24 hours of acute stroke (219 patients) was associated with an increased hazards ratio of 2.41 (95\% CI: 1.24-4.67) for long-term mortality after adjustment for multiple variables, compared to the reference group (140-159 $\mathrm{mmHg}$ ). Ahmed's study ${ }^{15}$ showed that the odds ratio for combined death or dependency at Day 21 of stroke onset was significantly higher in patients with high initial BP than that in those with normal initial BP in multiple logistic regression analyses. Some observational studies in western populations found a U-shaped association $^{16,17}$, such that extreme low and high BP values were associated with poor outcome after stroke onset. Others did not find a significant association between admission BP and poor outcome among acute ischemic stroke patients ${ }^{20,21,25}$. Aslanyan's study ${ }^{20}$ showed that baseline MAP was not associated with poor ischemic stroke outcome. However, variables describing the course of BP over the first 2.5 days had a marked and independent relationship with one and three month outcome. The effect of BP reduction among acute stroke patients is much less certain $^{11,26}$. For many years there has been argument regarding whether to lower or to raise BP and whether to continue or to stop antihypertensive treatment among acute stroke patients ${ }^{11}$. The best way to answer this question is to carry out randomized clinical trials. Eveson et $\mathrm{al}^{27}$ conducted a pilot study about effects of lowering BP among acute ischemic stroke patients. In their pilot study, a total of 40 hypertensive acute ischemic stroke patients were randomized to a lowered BP group $(\mathrm{n}=18)$ who received treatment of lowered BP for 14 days and control group $(n=22)$, and stroke outcome measures were assessed at Day 14. Their findings showed that neurologic and functional measures were similar between groups. Recently, Potter and colleagues ${ }^{28}$ published the findings of their pilot trial on controlling hypertension and hypotension immediately post-stroke (CHHIPS), among 179 patients with cerebral infarction or cerebral haemorrhage and hypertension. Their findings showed that there was no evidence of early neurological deterioration with active lowering BP treatment despite the significantly greater fall in systolic BP within the first 24 hours in lowering BP group compared with placebo. However, three month mortality was much lower in lowering BP group compared with control group. It must be stressed that the two pilot studies are of small sample, a large randomized clinical trial still needs to be conducted to determine the effects of lowering BP on clinical outcome for acute ischemic stroke.

There is evidence that cerebral blood flow becomes dependent on systemic BP after a loss of cerebrovascular autoregulation and that insufficient cerebral perfusion pressure through the ischemic penumbra may increase the risk of neurological damage ${ }^{29,30}$. However, in a meta-analysis ${ }^{31}$ which assessed the effect of antihypertensive agents on cerebral blood flow and flow velocity, the authors believed that there was little existing evidence that antihypertensive agents reduce cerebral blood flow in spite of their effects of lowering BP.

Acute phase BP recorded in our study, and in other observational studies, might reflect the pathophysiological responses to acute ischemic stroke and the severity of disease. Our study was limited in that it was an observational study. Only a randomized control clinical trial on BP treatment can determine the causal effect of lowering BP on mortality and neurologic deficiency among acute ischemic stroke patients ${ }^{11}$.

\section{Conclusion}

In summary, our study found that systolic $\mathrm{BP} \geq 180 \mathrm{mmHg}$ and diastolic $\mathrm{BP} \geq 120 \mathrm{mmHg}$ were significantly and positively associated with death in-hospital or NIHSS $\geq 10$ at discharge among patients with acute ischemic stroke. This study suggests that a large randomized controlled clinical trial is needed to test the potential beneficial effect of lowering BP in acute ischemic stroke patients. 


\section{REFERENCES}

1. WHO. Global burden of disease 2002; deaths by age, sex and cause for the year 2002. Geneva, Switzerland: World Health Organization; 2003.

2. Yusuf S, Reddy S, Ounpuu S, Anand S. Global burden of cardiovascular diseases. Part II: variations in cardiovascular disease by specific ethnic groups and geographic regions and prevention strategies. Circulation. 2001;104(23):2855-64.

3. He J, Gu D, Wu X, et al. Major causes of death among men and women in China. N Engl J Med. 2005;353(11):1124-34.

4. Wei G, Ji X, Bai H, Ding Y. Stroke research in China. Neurol Res. 2006;28(1):11-15.

5. Zhang LF, Yang J, Hong Z, et al. Proportion of different subtypes of stroke in China. Stroke. 2003;34(9):2091-6.

6. Hajat C, Dundas R, Stewart JA, et al. Cerebrovascular risk factors and stroke subtypes. Stroke. 2001;32(1):37-42.

7. Prospective Studies Collaboration. Cholesterol, diastolic blood pressure, and stroke: 13000 strokes in 450,000 people in 45 prospective cohorts. Lancet. 1995;346(8991-2):1647-53.

8. Eastern Stroke and Coronary Heart Disease Collaborative Research Group. Blood pressure, cholesterol, and stroke in eastern Asia. Lancet. 1998;352(9143):1801-7.

9. Whelton PK, He J. Blood pressure reduction. In: Manson JE, Buring JE, Ridker PM, Gaziano JM, editors. Clinical trials in cardiovascular disease, 2nd ed. Philadelphia: Elsevier Saunders; 1999. p. 282-96.

10. Bath P, Chalmers J, Powers W, et al. International Society of Hypertension Writing Group. International Society of Hypertension (ISH): statement on the management of blood pressure in acute stroke. J Hypertens. 2003;21(4):665-72.

11. Chalmers J. Blood pressure in acute stroke: in search of evidence. J Hypertens. 2005;23(2):277-8.

12. Britton M, Carlsson A. Very high blood pressure in acute stroke. J Intern Med. 1990;228(6):611-5.

13. Dandapani BK, Suzuki S, Kelley RE, Reyes-Iglesias Y, Duncan RC. Relation between blood pressure and outcome in intracerebral haemorrhage. Stroke. 1995;26(1):21-4.

14. Robinson TG, Dawson SL, Ahmed U, Manktelow B, Fortherby MD, Potter JF. Twenty-four hour systolic blood pressure predicts long-term mortality following acute stroke. J Hypertens. 2001; 19(12):2127-34

15. Ahmed N, Wahlgren N. High initial blood pressure after acute stroke is associated with poor functional outcome. J Intern Med. 2001;249(5):467-73.

16. Leonardi-Bee J, Bath PM, Phillips SJ, Sandercock PA. Blood pressure and clinical outcomes in the International Stroke Trial. Stroke. 2002;33(5):1315-20.

17. Okumura K, Ohya Y, Maehara A, Wakugami K, Iseki K, Takishita S. Effects of blood pressure levels on case fatality after acute stroke. J Hypertens. 2005;23(6):1217-23.
18. Vemmos KN, Tsivgoulis G, Spengos K, et al. U-shaped relationship between mortality and admission blood pressure in patients with acute stroke. J Intern Med. 2004;255(2):257-65.

19. Castillo J, Leira R, Garcia M, Serena J, Blanco M, Davalos A. Blood pressure decrease during the acute phase of ischemic stroke is associated with brain injury stroke outcome. Stroke. 2004;35(2):520-6

20. Aslanyan S, Fazekas F, Weir CJ, Horner S, Lees KR. Effect of blood pressure during the acute period of ischemic stroke on stroke outcome: atertiary analysis of the GAIN International Trial. Stroke. 2003;34(10):2420-5.

21. Carlberg B, Asplund K, Hagg E. The prognostic value of admission blood pressure in patients with acute stroke. Stroke. 1993;24(9): 1372-5.

22. Perloff D, Grim C, Flack J, et al. Human blood pressure determination by sphygmomanometry. Circulation. 1993;88(5 Pt 1):2460-70.

23. Allain CC, Poon LS, Chan CS, Richmond W, Fu PC. Enzymatic determination of total serum cholesterol. Clin Chem. 1974;20 (4):470-5.

24. Friedewald WT, Levy RI, Fredrickson DS. Estimation of the concentration of low-density lipoprotein cholesterol in plasma, without use of the preparative ultracentrifuge. Clin Chem. 1972; 18(6):499-502.

25. Willmot M, Leonardi-Bee J, Bath PM. High blood pressure in acute stroke and subsequent outcome: a systematic review. Hypertension. 2004;43(1):18-24.

26. Bath P, Chalmers J, Powers W, et al. International Society of Hypertension Writing Group. International Society of Hypertension (ISH): statement on the management of blood pressure in acute stroke. J Hypertens. 2003;21(4):665-72.

27. Eveson DJ, Robinson TG, Potter JF. Lisinopril for the treatment of hypertension within the first 24 hours of acute ischemic stroke and follow-up. Am J Hypertens. 2007;20(3):270-7.

28. Potter JF, Robinson TG, Ford GA, et al. Controlling hypertension and hypotension immediately post-stroke (CHHIPS): a randomised, placebo-controlled, double-blind pilot trial. Lancet Neurol. 2009;8(1):48-56.

29. Dawson SL, Blake MJ, Panerai RB, Potter JF. Dynamic but not static cerebral autoregulation is impaired in acute ischaemic stroke. Cerebrovasc Dis. 2000;10(2):126-32.

30. Robinson TG, James M, Youde J, Panerai R, Potter J. Cardiac baroreceptor sensitivity is impaired after acute stroke. Stroke. 1997;28(9):1671-6.

31. Sare GM, Gray LJ, Bath PM. Effect of antihypertensive agents on cerebral blood flow and flow velocity in acute ischaemic stroke: systematic review of controlled studies. J Hypertens. 2008;26(6):1058-64. 